Entrevista

\section{Conexiones Americanas. Una conversación con Montserrat Ventura i Oller}

JORGE MOREIRA PEÑA ${ }^{1}$

(D) 0000-0003-1122-6737

Universitat Autònoma de Barcelona, España

CLAUDIA SOTO GARCÍA ${ }^{2}$

0000-0003-1468-9235

Universitat de Barcelona, España

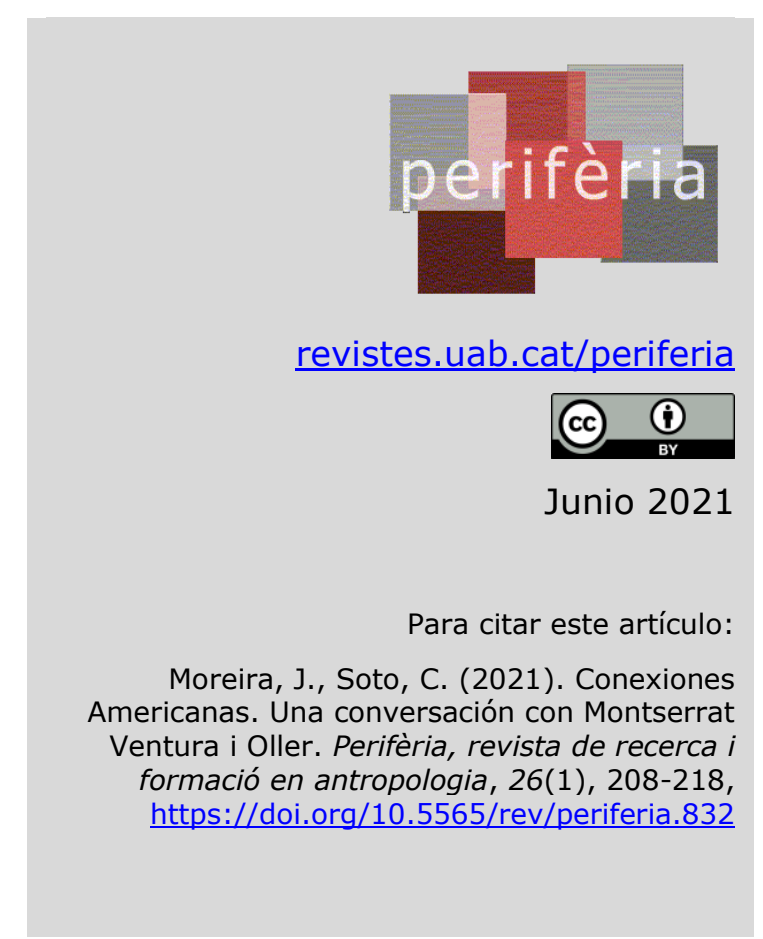

\title{
Resumen
}

En el marco del proyecto radiofónico Conexiones Americanas: Hacia los Ríos Profundos de América Latina, invitamos a la Dra. Montserrat Ventura i Oller a dialogar sobre su experiencia como investigadora y sus conocimientos de la sociedad Tsachila. En esta conversación conocemos detalles de sus intereses y perspectivas de análisis, así como de los cambios que ha podido observar en la sociedad Tsachila desde que realiza trabajo de campo. En ella también nos recuerda la importancia de la Antropología para buscar, con humildad, el conocimiento profundo de la diversidad humana.

Palabras clave: Etnografía; Antropología histórica; Ontologías indígenas; Identidades sociales; Sociedad Tsachila.

\section{Abstract: American Connections. A conversation with Montserrat Ventura $i$ Oller}

Within the framework of the radio project American Connections: Towards the Deep Rivers of Latin America, we invited PhD. Montserrat Ventura i Oller to discuss her

\footnotetext{
${ }^{1}$ Contacto: Jorge Moreira Peña - jorge.moreira.p@hotmail.com

${ }^{2}$ Contacto: Claudia Soto García - claudiasotogarcia@gmail.com
} 
experience as a researcher and her knowledge of Tsachila society. In this conversation, we learn details of his interests and perspectives for analysis, as well as the changes that she has been able to observe in Tsachila society since she has done fieldwork. In it, she also reminds us of the importance of Anthropology to seek, with humility, a deep knowledge of human diversity.

Keywords: Ethnography; Historical anthropology; Indigenous ontologies; Social Identities; Tsachila society.

\section{Introducción al programa}

Desde el Colectivo de Estudios Latinoamericanos de Barcelona (CELAB) el año 2019 iniciamos un proyecto radiofónico, dedicado a dialogar sobre los pueblos indígenas de Latinoamérica: Conexiones Americanas: Hacia los Ríos Profundos de América Latina $^{3}$. En este proyecto nos propusimos como objetivo amplio mostrar la enorme diversidad cultural del continente americano, y como objetivo específico contribuir a la divulgación del conocimiento en referencia a los pueblos indígenas. El proyecto consistió en un ciclo de entrevistas a especialistas de distintas áreas de las Ciencias Sociales, las que eran grabadas en el estudio radiofónico de UAB Campus Mèdia, motivo por el que en marzo del año 2020 tuvo que ser suspendido por cierre de las dependencias de la universidad debido al decreto de estado de alarma en el país en consideración al COVID-19. En uno de aquellos programas invitamos a la Dra. Montserrat Ventura para dialogar de sus investigaciones sobre la sociedad Tsachila, quien amablemente aceptó nuestra invitación.

Quisiéramos aclarar que la entrevista fue realizada en formato oral y no estuvo pensada en una publicación escrita como la que aquí presentamos. Sin embargo, hemos querido hacer el traspaso de la conversación sostenida con Montserrat al medio escrito por considerar que contiene muchos elementos significativos que hacen

\footnotetext{
${ }^{3}$ El programa ha querido brindar un homenaje a José María Arguedas, cuyo nombre ha sido inspirado en el título de su tercera novela, Los Ríos Profundos, que en 1958 fue publicada por la Editorial Losada. La introducción al programa, donde presentamos nuestro manifiesto, y la entrevista a Montserrat Ventura se encuentran en los siguientes enlaces:

https://campusmedia.uab.cat/index.php/video/1970/conexiones-americanas-t02e01/ https://campusmedia.uab.cat/index.php/video/2051/conexiones-americanas-t02p04/
} 
deseable y pertinente una mayor difusión -a pesar del limitado tiempo disponible en el programa y que restringe su profundización (30 minutos)-: los detalles de su trayectoria personal, de su trabajo de campo, de su enfoque antropológico que conjuga perspectivas históricas y ontológicas, entre otros temas. En vista que la oralidad tiene una dinámica distinta a la escritura, con Montserrat hemos optado por realizar leves modificaciones en orden a facilitar la lectura, sin alterar en absoluto el contenido de la conversación. A continuación, transcribimos la entrevista como se desarrolló en el estudio de grabación el mes de noviembre del año 2019 y que contiene la presentación a la Dra. Montserrat Ventura.

\section{La entrevista}

Jorge Moreira: Bienvenidos a este nuevo programa de Conexiones Americanas, un proyecto del Colectivo de Estudios Latinoamericanos de Barcelona, que en esta segunda temporada se estará dirigiendo Hacia los Ríos Profundos de América Latina, pues estaremos centrados en conversaciones sobre y en colaboración con los pueblos indígenas de América. En esta oportunidad tenemos la fortuna de conversar con Montserrat Ventura i Oller, quien es Doctora en Antropología por la Escuela de Estudios Superiores en Ciencias Sociales de Paris (EHESS), actualmente es directora del Departamento de Antropología Social y Cultural de la Universitat Autònoma de Barcelona ${ }^{4}$, donde además ha coordinado el grupo de investigación Antropología e Historia de la construcción de identidades sociales y políticas (AHCISP). Ella ha realizado sus investigaciones en las tierras bajas de Ecuador, donde se puede decir que ha conducido un estudio longitudinal, ya que lleva más de 20 años realizando trabajo de campo con la sociedad Tsachila, en el que algunos de sus temas de interés han sido el chamanismo, la ritualidad, la cosmología y la construcción de las identidades sociales. Para ejemplificar esto podemos decir que el año 2018 se publicó un monográfico en la Revista de Antropología Iberoamericana de la AIBR coordinado por Montserrat y que llevó por título: Humanidades amerindias en transformación:

\footnotetext{
${ }^{4}$ Al momento de la entrevista Montserrat se desempeñaba como directora del Departamento, cargo que dejó en septiembre de 2020.
} 
ontologías, dinamismo y contextos. Y recientemente, también el año 2018, se ha publicado por Edicions Bellaterra un libro del que es co-editora y que lleva por nombre: Humanidad. Categoría o condición. Un viaje antropológico. Muchas gracias Montserrat por estar con nosotros, por aceptar esta invitación.

Montserrat Ventura: Hola, muchas gracias a vosotros por invitarme. Me gustaría empezar por saludar esta iniciativa y agradecer a Jorge y a Claudia por su excelente labor. Comentabas que he coordinado el grupo AHCISP, Antropología e Historia de la construcción de identidades sociales y políticas, que actualmente coordina Josep Lluís Mateo. Es un grupo que fue fundado por la profesora Verena Stolcke en el Departamento de Antropología Social y Cultural de la UAB, del que todavía es miembro bien activa y muy inspiradora en toda nuestra labor, y es por esto que nosotros trabajamos antropológicamente con una mirada también histórica, y miramos la historia con una perspectiva antropológica. Efectivamente, yo empiezo mi formación en la Universidad Autónoma de Barcelona en Antropología, pero bien rápido aquí aprendo que la Antropología se ha debatido en dos paradigmas, el paradigma de la unidad del ser humano, en el que hay que insistir para no caer en la desigualdad, y el paradigma de buscar la diversidad, de entender y comprender la diversidad, de esta riqueza humana que tenemos. Entonces, como decía la profesora Verena Stolcke "en toda teoría hay algo de biografía", y yo creo que hay una parte significativa de la Antropología catalana que ha sido especialmente sensible a la cuestión de la diversidad, por el contexto en que vivimos, y este fue realmente mi punto de partida. Es así como yo busco en mis estudios intentar entender la diversidad, probablemente la más lejana posible, de ahí mi interés por sociedades de las tierras bajas de América.

J: ¿Cómo es que llegas allá? ¿Cómo tomas la decisión de ir a América, de ir a Ecuador?

M.V: Yo me interesé más por las sociedades amazónicas en un principio, no tanto América en general como la Amazonía precisamente, porque era el paradigma de la sociedad salvaje, la otredad. Y aquí en Barcelona había especialistas en América Colonial, en la América Andina, en Mesoamérica, pero no había especialistas en Amazonía. Entonces busco esta especialidad en Francia, es así que me fui a París y allí Philippe Descola -que en aquel momento era un joven profesor, creo que fui una de sus primeras alumnas, no la primera en graduarme, pero sí la primera en llegar- 
era especialista en Amazonía, había trabajado en una sociedad, la Achuar, de la Amazonía ecuatoriana. Digamos que fue un punto de partida por los contactos, una cuestión práctica, él tenía muchos contactos en Ecuador y es así como llego a este lugar.

J: Sin embargo, tú no estudias la Amazonía, sino que vas a un lugar de la Selva, hacia la costa del Pacífico.

M.V.: Efectivamente, él me da los contactos, y sabiendo ya que me dirijo al Ecuador yo busco qué estudios se han hecho, qué estudios faltan por hacer y me voy a Ecuador con tres posibilidades. Allí empiezo a hablar con los especialistas locales, que es algo que siempre se debería hacer, puesto que son ellos los que conocen la realidad local; me voy a ver profesores de Antropología de la Universidad Católica, de la FLACSO, básicamente de esas dos universidades; y me voy también a ver algunos misioneros que estaban trabajando en plena selva. Entonces de las tres posibilidades, parecía claramente que el grupo del que menos se sabía y que más urgencia había por estudiar -en aquel tiempo donde todavía se pensaba en urgencias por desaparición- era el Tsachila, que en aquel momento era conocido como Colorado.

J: Bueno, tú realizaste tu tesis doctoral con la sociedad Tsachila de la que resulta el texto En el cruce de caminos. Identidad, cosmología y chamanismo tsachila. ¿Nos podrías contextualizar la sociedad Tsachila que tú viste y estudiaste?

M.V.: Sí; las cosas van muy rápido, y la que yo descubrí en aquel momento no es la misma de ahora: efectivamente, en una generación, en este momento de contextos globales, ha sufrido muchos cambios. Bueno la Antropología siempre se ha topado con sociedades que aparentemente están sufriendo muchos cambios, porque las sociedades cambian, esta es la verdad. Entonces los Tsachila, antes llamados Colorados, vivían en lo que se conoce como Costa: en Ecuador se considera que hay tres grandes regiones geográficas: Selva, Costa y Amazonía. Ellos se ubican en el lado occidental de los Andes, tradicionalmente conocido como Costa, pero que no es litoral en absoluto, aunque sí son tierras bajas; y yo insisto en esto de las tierras bajas porque desde la perspectiva antropológica se trata de un tipo de sociedad muy parecida a la que habitualmente se ha descrito para la Amazonía -esto, desde la tradición, porque ahora, como decía, han cambiado-: eran sociedades pequeñas, que 
vivían en hábitat disperso, que son cazadores, recolectores, pescadores y agricultores, pero que practican agricultura itinerante; sociedades que son muy igualitarias, pero que, sin embargo, tienen una figura prominente que es el chamán y que es muy importante a nivel de toma de decisiones en el grupo. Insisto, desde la perspectiva tradicional. Son muy poquitos: en aquel tiempo eran alrededor de 2000, y ahora se calculan unos 1000 más. Y mantenían su lengua, esto también era determinante, porque yo iba allí intentando entender la identidad, que era el gran tema de la época también, porque yo llegué allí a principios de los años 90, con el boom de los estudios identitarios.

J: ¿Y tú realizas tu estudio también a partir del idioma, tú aprendiste tsafiki?

M.V.: Debo decir que las lenguas indígenas son muy sofisticadas y muy complejas de entender para un hablante de una lengua occidental, por varias razones. En primer lugar, porque somos del paradigma de la lectoescritura y éstas son lenguas de tradición oral, por lo que cuestan más de aprender; y luego porque son lenguas que antes se denominaban aglutinantes -los lingüistas ahora me reñirían mucho porque las descripciones son distintas-, pero que presentan una complejidad estructural que a nosotros nos resulta muy difícil de comprender. Yo intenté aprender pidiendo que me enseñaran con lo que yo sé hacer, lectoescritura: iba a clases con Ramón Aguavil, un investigador Tsachila de mi edad, en aquel entonces joven, y me ponía deberes que yo hacía. Pero sobre todo aprendí mucho con los niños, porque los niños no hablaban castellano, y con los adultos era más fácil pasarse a esta lengua. Tengo que reconocer que no empecé a comprender un poco mejor la lengua hasta que llegó allí una lingüista, Connie Dickinson ${ }^{5}$, quien ha hecho un trabajo magnífico a lo largo de casi 30 años, y ella pudo explicarme las complejidades de la lengua.

J: Hicieron un trabajo conjunto entonces.

M.V.: Sí, sin proponérnoslo; es decir, colaborábamos cada una haciendo su propia investigación. Aunque en un momento dado yo colaboré en uno de sus proyectos,

\footnotetext{
${ }^{5}$ Connie Dickinson, Dra. en lingüística por la Universidad de Oregon (2002), trabaja desde 1994 en la descripción y análisis del tsafiki y en un proyecto colaborativo de documentación de la lengua y la cultura Tsachila. Actualmente trabaja también en otras lenguas del Ecuador, como las del pueblo Chachi, Quichua Amazónico, Shuar y Wao.
} 
muy interesante, un trabajo colaborativo de documentación de la lengua y la cultura Tsachila ${ }^{6}$; era su proyecto pero yo participé formando en Antropología a un grupo de jóvenes Tsachila, preparando algunas entrevistas de temas antropológicos y elaborando algunas entradas del diccionario.

J: Volviendo al tema de la identidad, ¿nos puedes comentar algunos hallazgos que hayas encontrado respecto a la vinculación entre chamanismo e identidad en la sociedad Tsachila?

M.V.: Lo que dominaba en los años 80 y 90 era la consideración de la identidad desde la perspectiva sociológica, es decir, una perspectiva que se interesaba por los rasgos identitarios externos de los pueblos, y por las relaciones interétnicas, y al empezar a estudiar el chamanismo me di cuenta que era fundamental entender la identidad también desde una perspectiva más de lógicas sociales y de ontologías, y esto nos lo podía proporcionar el chamanismo: cómo era su visión del mundo, cómo se relacionaban los humanos con los no humanos, y los chamanes eran los especialistas de este contacto. Con el chamanismo aprendí dos cosas: que estas sociedades se relacionan de una forma muy particular, igualitaria, con los no humanos, pero también que el chamanismo es un punto de inflexión en sus relaciones con los otros humanos, porque el chamanismo se constituye en centro de una red de relaciones interétnicas muy relevante, que nos permite reconfigurar la idea de sociedades estáticas, aisladas en sí mismas, algo que no define en absoluto las sociedades indígenas.

J: Montserrat, antes de abordar el tema de las ontologías, te quería preguntar, como docente que llevas mucho tiempo trabajando y que actualmente eres directora del Departamento de Antropología, ¿Qué importancia se le otorga a la Antropología americanista desde el Departamento de la Universidad Autónoma de Barcelona, específicamente pensando en investigaciones sobre pueblos indígenas?

M.V.: Hay una cuestión importante aquí. En nuestro departamento, y creo que en general en la Antropología de aquí, de la catalana en todo caso, no hay la tradición

\footnotetext{
${ }^{6}$ Se trata del proyecto que forma parte del archivo DOBES de Documentation of Endangered Languages, albergado por el Max Plank Institute for Psycholinguistics (Holanda) https://dobes.mpi.nl/projects, y actualmente en el Archivo de Lenguas y Culturas del Ecuador, albergada por la FLACSO de este país https://flacso.edu.ec/lenguas-culturas/sobre-el-archivo/que-es/
} 
que encontramos en otros lugares, como por ejemplo en Francia donde me formé, de Departamentos de Antropología unificados a partir de áreas culturales. En general la Antropología o se rige por ámbitos temáticos o por ámbitos areales, son dos tradiciones distintas. Este último aquí no lo encontramos, por tanto no se podría decir que el americanismo es en sí constituyente de algún tipo de unidad. Aquí se ha trabajado más en líneas temáticas y es por esto que, por ejemplo, yo me ubico en un grupo de investigación donde hay especialistas de muchos lugares del mundo. Entonces si me preguntas qué lugar tiene el americanismo te diré: el americanismo se ha formado históricamente, como en todos los lugares, por una tradición colonial, el interés de España hacia América ha sido por su relación colonial; y el primer americanismo en Antropología se fijó en las culturas de las áreas de relación histórica, de las áreas andinas y mesoamericanas, y por tanto a nivel temático el interés era otro, era estudiar otras realidades vinculadas con estas sociedades. En el momento en que se introducen un poco más los estudios sobre la Amazonía, empieza a desarrollarse también el interés por otros campos, como el de las ontologías que comentábamos anteriormente. Uno de los centros a partir del cual surgen los análisis de la ontología ha sido la Amazonía, a partir de dos grandes antropólogos que son Philippe Descola, profesor del Collège de France, ahora recientemente jubilado, con su propuesta de los cuatro modos de relación y de identificación de referencia, y Eduardo Viveiros de Castro, profesor del Museo Nacional de Río de Janeiro, este último con el perspectivismo amerindio. Yo creo que estos dos paradigmas han revolucionado la forma de entender la Antropología desde la perspectiva ontológica.

J.: En ese sentido, considerando que estudiaste con Descola y tienes interés en perspectivas ontológicas, ¿te declaras discípula de Descola?

M.V.: Me declaro su discípula, porque él fue mi director de tesis, él dirigió mi tesis doctoral y yo lo he ido siguiendo. Es cierto que cuando me trasladé, al constituir el grupo de investigación (AHCISP), incorporé una mirada más cercana a la antropología histórica, que creo que me ha permitido cruzar dos perspectivas necesarias a la hora de comprender los pueblos indígenas: los contextos y las ontologías. Por un lado, la antropología histórica nos permite entender las relaciones de poder, las relaciones coloniales, y por otro lado, los estudios de la ontología nos permiten entender las lógicas: los primeros sin las segundas no se pueden entender, y viceversa. Si buscamos solo contextos anulamos la agencia indígena, su capacidad de entender el 
mundo de formas distintas, porque fijarse solo en los contextos es otorgar de alguna forma todo el poder de decisión al exterior. Y si por otro lado anulamos los contextos y solo nos centramos en una ontología estamos hablando de realidades fuera del mundo, estáticas, y las realidades, también de las sociedades indígenas, son procesuales. Entonces yo creo que esta doble mirada nos permite entender los pueblos indígenas, y desde nuestro grupo de investigación es lo que estamos intentando hacer.

J: Uno de los últimos proyectos que ustedes han realizado se llama Hum-Ant: Estudio antropológico comparativo de las nociones de ser humano, en el que está implícito el cuestionamiento a la ontología moderna, más cientificista, que declara esta separación entre la naturaleza y la cultura, para abrirse a la posibilidad de que hay múltiples realidades, que hay múltiples mundos. ¿Qué nos puedes decir de los resultados que ustedes han obtenido de esta investigación comparativa de la idea de humanidad?

M.V.: Yo creo que una de las virtudes de este grupo de investigación es tener especialistas de distintos lugares y de distintos momentos históricos -que esto es relevante también-, lo que nos ha permitido entender algo que ya suponíamos en todo caso, que la noción de ser humano no es universal, a pesar de que el ser humano es igual en todas partes y a la vez diverso, pero que no todas las sociedades tienen la misma noción del ser humano. Mientras que el racionalismo científico -que no es el único paradigma en occidente, puesto que hay otros paradigmas ontológicos que conviven con el científico, y no todos pensamos siempre en términos científicos, pero sí es la lógica que se puede tomar como dominante aquí-, se basa en lo que Philippe Descola llama naturalismo -esta división radical entre naturaleza y cultura-, en cambio en otras sociedades dominan otras ontologías; pongamos el ejemplo de una ontología opuesta como el animismo propio de las sociedades amazónicas, y también, por qué no, de la Tsachila, que entiende que hay una continuidad entre humanos y no humanos, de forma que todos los seres parten de una humanidad de base, con apariencias distintas, y que esta humanidad se manifiesta en grados distintos -por descontado-, y que cada sociedad otorga importancia a uno u otro elemento necesario para constituir el ser humano, por ejemplo las formas de comunicación, que es uno de los elementos esenciales marcadores de la condición humana en esta ontología. 
J: En ese sentido, pensando en la comunicación, ¿de qué manera podemos llegar a uniones, a comunicaciones fructíferas? Te pregunto porque las ontologías nos demuestran que hay un problema en la comunicación entre distintas culturas. Cuesta hacer una traducción, por ejemplo, de la ontología Tsachila, que permita comunicarse con una ontología distinta, que podría ser la naturalista. ¿Cómo se puede llegar a estos puntos en común para que los seres humanos, en este planeta que habitamos, logremos entendernos y respetarnos en igualdad de condiciones?

M.V.: Te agradezco la pregunta, porque insistir en las características distintivas de cada sociedad a veces produce una reacción opuesta a la que se pretende -de decir no hay comprensión mutua posible-, y se trata de todo lo contrario. Lo que sí es cierto es que hace falta conocimiento y un poco de humildad. Las sociedades indígenas nos han demostrado esa humildad, esta capacidad de intentar entender estas formas y estos paradigmas que no le eran propios. A través de los estudios universitarios, a través del contacto cotidiano que tienen necesariamente con este otro paradigma, ellos lo llegan a comprender. Es genial cómo hablas con personas que surgen de esa otra tradición indígena y ellos te van intentando traducir para que tú entiendas; por ejemplo, cuando los chamanes me intentaban explicar su universo y me decían "esto es como cuando ustedes se gradúan en la universidad...", yo pensaba "qué genial ¿no?". En cambio, a nosotros nos cuesta mucho más intentar buscar estos elementos comparativos para hacer el mismo proceso a la inversa. Entonces yo creo que, si desde ambas partes hubiera esta humildad, que pasa imprescindiblemente por el conocimiento del otro, por el conocimiento profundo del otro, no superficial, el diálogo sería más igualitario y fructífero, y yo creo que aquí la Antropología tiene un gran rol.

J: Montserrat, tú tienes muchas publicaciones, tú conoces en profundidad la sociedad Tsachila. Respecto a tus investigaciones chas realizado trabajos de conversación con la propia sociedad Tsachila para entregar resultados, para ir dialogando lo que tú investigas y tratar de contraponerlo, o mostrarlo para que ellos vayan viendo lo que se está pensando de su sociedad?

M.V.: Yo he realizado un trabajo de campo desde los años 90, y en esos años había mucha menos gente formada en el sistema escolar, mucha gente formada y capaz, pero no en el sistema escolar, por ejemplo, en la lectoescritura. Recuerdo que en mi primer texto pedí al que entonces era mi profesor de tsafiki, cuyo criterio sigue siendo 
para mí un referente, que lo leyera por si veía algo inconveniente o algún error antes de publicarlo, y yo esperando impaciente, porque me lo pedían los editores, y al cabo de 3 o 4 meses al insistirle me dijo "Sí, sí, ya empecé, voy por la primera página..."; realmente en aquel momento era más complejo compartir los textos, era más fácil oralizar las ideas: "Mire ¿qué le parece esto?" Porque al final su comentario se limitó a constatar: "Usted utiliza mucho el término Colorados y nosotros nos llamamos Tsachi", y es que era un texto que hablaba de la historia y yo usaba documentos de archivo. Ahora es mucho más fácil socializar los resultados; es más, ahora hay muchos más jóvenes que están investigando ellos mismos sobre su propia realidad y documentándola. Claro, entre medio lo que ha habido son personas que se han formado por su cuenta, y una interacción, de muchos años, como de este profesor de tsafiki y muchos otros Tsachila con la lingüista Connie Dickinson, con el proyecto de documentación, conmigo, con otros investigadores, y ha habido muchas ONGs, muchos proyectos gubernamentales, unos cambios enormes... ahora hay mujeres que están en la vida política pública, lo que antes era impensable, o por ejemplo este grupo de investigación local dirigido por Alfonso Aguavil, que se llama Pikitsa7, que creo está haciendo una tarea enorme de documentar su propia cultura. Entonces la comunicación parece ahora más fluida. Simplemente han pasado muchas cosas entre tanto, no solo la Antropología.

\section{Bibliografía}

Ventura, M. (2018). Humanidades amerindias en transformación: ontologías, dinamismo y contextos. Presentación al monográfico Humanidades amerindias en transformación. AIRB. Revista de Antropología Iberoamericana, 13(2), 153168. https://doi.org/10.11156/aibr.v13i2.68514

Ventura, M., Mateo, J. L. \& Clua, M. (Eds.). (2018). Humanidad. Categoría o Condición. Un viaje antropológico. Barcelona: Edicions Bellaterra.

Enlace a entrevista de Montserrat Ventura i Oller disponible en UAB Campus Mèdia: https://campusmedia.uab.cat/index.php/video/2051/conexiones-americanast02p04/

\footnotetext{
7 Alfonso Aguavil es fundador de Pikitsa y colaborador del Archivo de Lenguas y Culturas del Ecuador https://flacso.edu.ec/lenguas-culturas/colaboradores/alfonso-aguavil-calazacon/
} 